\title{
Prevalence and Impact of Fibrinolytic Dysregulation in Patients with Acute Coronary Syndromes
}

Huaibin Wan ( $\nabla$ docvanhb@outlook.com )

Dongguan people's hospital https://orcid.org/0000-0001-7962-0939

\section{Zhihao Wu}

Dongguan People's Hospital

Zhenbang Lie

Dongguan People's Hospital

\section{Daqiang Li}

Dongguan People's Hospital

\section{Shaohui Su}

Dongguan People's Hospital

\section{Research article}

Keywords: fibrinolysis, thrombelastography, acute coronary syndrome, retrospective study

Posted Date: June 4th, 2020

DOI: https://doi.org/10.21203/rs.3.rs-32637/v1

License: (c) (1) This work is licensed under a Creative Commons Attribution 4.0 International License. Read Full License 


\section{Abstract}

BackgroundXDual antiplatelet therapy can reduce coronary thrombosis and improve the prognosis in patients with acute coronary syndrome (ACS). However, there was limited prognostic information about fibrinolytic dysregulation in patients with ACS. This study is aimed to evaluated the prevalence and impact of fibrinolytic dysregulation in patients with acute coronary syndrome (ACS).

Methods $₫$ We retrospectively analyzed coagulation and fibrinolysis related indexes of ACS in hospitalized adults with rapid thrombelastography between May 2016 and December 2018. All of the follow-up visits were ended by December 2019. The primary outcome was the occurrence of major adverse cardiovascular events (MACEs), which included unstable angina pectoris, non-fatal myocardial infarction, non-fatal cerebral infarction, heart failure and all-cause death.

Results $₫ 338$ patients were finally included with an average age of $62.5 \pm 12.8$ years old, $273(80.5 \%)$ were males, 137(40.5\%) patients were with STEMI. Fibrinolysis shutdown and hyperfibrinolysis were observed among 163 (48.2\%) and 76(22.5\%) patients, respectively. During a total of 603.2 person-years of followup period, 77 MACEs occurred (22.8\%). Multivariate Cox regression analysis indicated that age [HR: 1.031 $95 \% \mathrm{Cl}: 1.007-1.056, \mathrm{P}=0.012]$ and LY30 [HR: $1.097,95 \% \mathrm{Cl}: 1.013-1.188, \mathrm{P}=0.023]$ were independently correlated with the occurrence of MACEs. The hazard ratios pertaining to MACEs in patients with LY30X $0.8 \%$ and $₫ 3.0 \%$ compared with those in the physiologic range(LY30: 0.8-3.0\%) were 2.275 [HR: 2.275, $95 \% \mathrm{Cl}: 1.241-1.241, \mathrm{P}=0.003$ ] and 1.196 [HR: 1.196, 95\% Cl: 0.679-2.109,P=0.535], respectively.

Conclusions: Fibrinolytic dysregulation is very common in selected patients with ACS, and hyperfibrinolysis (HF) (LY30 >3\%) is associated with poor outcomes in patients with ACS

\section{Introduction}

Acute coronary syndrome (ACS), a critical disease in cardiovascular system with high morbidity and mortality, is a kind of spontaneous in situ thrombotic disease in atherosclerotic coronary arteries[1]. Therefore, antithrombotic therapy has become a key strategy of the secondary prevention of coronary heart diseases (CHDs). Evidence-based studies have confirmed that dual antiplatelet therapy based on aspirin and $\mathrm{P} 2 \mathrm{Y} 12$ receptor antagonists can reduce coronary thrombosis and improve the prognosis of patients with ACS.[2] However, there was limited prognostic information about coagulation and fibrinolysis in patients with ACS.

Thromboelastography (TEG) is a commonly utilized test to evaluate the severely injured trauma patients $[3,4]$, which can continuously monitor the whole process of clotting, including the activation of platelet, coagulation and fibrinolysis of the dynamic change. Admission rapid TEG data can predict inhospital thromboembolic events[5] and guide volume resuscitation[6], and provide more useful and costeffective evaluation of the coagulation system than multiple conventional coagulation tests.[7, 8] Notably, either TEG detected hyperfibrinolysis or fibrinolysis shutdown was related to poor prognosis in patients with trauma or severe diseases.[9-12] In patients with CHDs, modified TEG was applied to 
measure platelet reactivity[13, 14] and coagulation function[15]. However, little is known about abnormal fibrinolysis in patients with CHDs. In this study, we observed the prevalence profiles of abnormal fibrinolysis and evaluated the relationship between coagulation and fibrinolysis related parameters and clinical outcomes in patients with ACS.

\section{Methods}

\section{Study population}

This was a retrospective analysis of ACS in adults presenting to Dongguan people's hospital with rapid TEG. We retrieved the medical electronic medical records (EMR) of total 12,754 inpatients between May 2016 and December 2018. ACS included either acute ST-elevation myocardial infarction (STEMI) or non ST-elevation ACS(NSTEACS) and was defined according to present guidelines[16, 17]. The inclusion criteria were: aged 18 years or older, males or females; patients with ACS receiving a loading dose of aspirin $300 \mathrm{mg}$ and clopidogrel $300 \mathrm{mg}$, and then $100 \mathrm{mg}$ and $75 \mathrm{mg}$ daily respectively; received a TEG test before coronary revascularization procedure; provided a written informed consent. The exclusive criteria were: patients who took clopidogrel irregularly or were intolerable to clopidogrel; serious infection; liver and/or coagulation dysfunction, and primary or acquired thrombocytopenia. Two senior cardiovascular specialists independently reviewed the correctness of the diagnosis according to the above criteria, and the disputed results were jointly discussed and decided by the members of the research team. The protocol was approved by the ethics committee of Dongguan people's hospital before conducting.

\section{Data collection}

Data collection included the patient's gender, age, risk factors or medical history, such as the history of smoking, hypertension, diabetes mellitus, cerebral infarction, CKDs, atrial fibrillation, heart failure and details of medication. Blood pressure, heart rate, height and weight were measured. The blood routine test (Advia2120, Siemens, Germany), alanine aminotransferase (ALT), serum creatinine and total cholesterol (Backman, USA), activated partial thromboplastin time(APTT), prothrombin time(PT), D-dimers and fibrinogen (STAGO STAR, France) were tested according to the standardized operation process of our hospital during the first visit.

\section{Thromboelastography}

The fasting antecubital venous whole blood samples was collected by 1:9 sodium citrate tubes (Jingz, Nanchang, China) from patients with ACS at least 6 hours after taking loading doses of clopidogrel $300 \mathrm{mg}$ and aspirin $300 \mathrm{mg}$. The samples were processed by TEG5000 coagulation analyzer (Haemonetics management Co., Ltd., Shanghai, China) according to manufactory's instruction, and tested within 2 hours of collection. All laboratories were processed by our hospital's central laboratory. The following parameters were obtained from the tracings of the TEG: R time (time to clot initiation), Ktime (total clotting time), angle (clot kinetics), maximal amplitude (MA, strength of the clot), LY30 (lysis 30 minutes after MA), adenosine diphosphate inhibition rate (ADP-IR) and arachidonic acid inhibition rate 
(AA-IR). Patients were categorized according to their fibrinolytic phenotypes as determined by their LY30, as follows: hyperfibrinolysis was defined as LY30 >3\%, fibrinolysis shutdown as LY30 of less than $0.8 \%$ and physiologic fibrinolysis as LY 30 between $0.8 \%$ and $3 \%$, based on the previous studies. [18-21]

\section{Follow-up and outcomes}

We firstly retrieved the follow-up information from our outpatient, emergency and rehospitalization records in the EMR system. If a corresponding visit record could not be obtained, researchers would perform a telephone interview. All of the follow-up visits were ended by December 2019. The primary outcome was major adverse cardiovascular events (MACEs), which included unstable angina pectoris, non-fatal myocardial infarction, non-fatal cerebral infarction, heart failure and all-cause death. Also, other leading to hospitalized adverse events, such as major bleeding, which was defined as bleeding associated with a reduction in hemoglobin of more than $20 \mathrm{~g} / \mathrm{L}$ or leading to a transfusion of more than 2 units of blood or packed cells or symptomatic bleeding into a critical area or organ, during the follow-up period were recorded.

\section{Statistical Analysis}

Continuous variables were reported as mean \pm standard deviation if homogeneous, or as median (interquartile range). We used analysis of variance to compare continuous variables among groups. Categorical variables were reported as counts (percentages) and compared with Pearson chi-square test (or fisher exact test). The risk factors of the MACEs were analyzed and carried out by time-to-first event analysis using a Cox regression model. A forward / stepwise regression method was used for multiple factors regression, $P<0.05, P>0.10$. The correlators of $L Y 30$ were analyzed by pearson bivariate correlation analysis. The risk of MACEs was descripted by the Kaplan-Meier curve. All hypothesis tests were 2-sided and carried out at a significance level of 0.05. Analyses were performed with SPSS 22.0 software package for Windows (SPSS, IBM, USA).

\section{Results}

During the period between May 2016 and December 2018, 490 patients with ACS had at least once TEG test (Figure1). According to the inclusion criteria and exclusion criteria, 338 patients with ACS were finally included in the study with an average age of $62.5 \pm 12.8$ years old, $272(80.5 \%)$ were males, $137(40.5 \%)$ patients were with STEMI. According to the result of LY30, fibrinolysis shutdown was the most common phenotype (163 cases, $48.2 \%$ ), followed by physiologic (99 cases, $29.3 \%$ ) and hyperfibrinolysis (77cases, 22.5\%). There were elevated serum creatinine and total cholesterol, reduced R- and K-time, and increased angle and AA-IR in patients with hyperfibrinolysis. Table 1 lists the clinical characteristics of the patients included in this study.

21 patients (6.2\%) were lost to follow-up during the first year. Our median follow-up period was 708 days (interquartile range: 323 to 1037 days). During a total of 603.2 person-years of follow-up period, 77 MACEs occurred (22.8\%), including ischemic cardiovascular events $35(10.4 \%)$, heart failure 24 (7.1\%), 
TIA or non-fatal cerebral infarction $26(7.7 \%)$, and all-cause death 17 (5.0\%), respectively. 9(2.7\%) bleeding events were observed. Table 2 lists the clinical outcomes in details. There was an increasing trend of MACEs in patients with hyperfibrinolysis $(P=0.057)$. Compare to physiologic state $(3 \%)$, stroke or TIA was significantly increased in settings of fibrinolysis dysregulation(shutdown $8 \%$, hyperfibrinolysis $13.2 \%, P=0.044)$.

Univariate Cox regression analysis showed that the occurrence of MACEs was significantly correlated with age[HR:1.043, 95\%Cl:1.022-1.063,P®0.001], systolic blood pressure[HR:1.009, 95\% Cl:1.0001.019,P=0.045], hemoglobin[HR:0.984, 95\% Cl:0.974-0.993,P=0.001], serum creatinine [HR:1.002, 95\% Cl:1.001-1.003, $P=0.003$ ], D-dimers [HR:1.147, 95\% Cl:1.024-1.285, $P=0.018]$,fibrinogen[HR: 1.193, 95\% Cl:1.029-1.385,P=0.020], Ly30[HR:1.117, 95\% Cl:1.041-1.198,P=0.002], but not with gender, BMI, medical histories (such as smoking, hypertension and diabetes), heart rate, leukocytes, platelets, total cholesterol, ALT, PT, APTT , R time, $\mathrm{K}$ time, angle, MA, ADP-IR and AA-IR. Multivariate Cox regression analysis based on age, gender, levels of systolic blood pressure, hemoglobin, serum creatinine, D-dimers, fibrinogen and LY30 indicated that age [HR: $1.03195 \% \mathrm{Cl}: 1.007-1.056, \mathrm{P}=0.012]$ and LY30 [HR: 1.097, 95\% Cl: 1.013$1.188, P=0.023]$ were independently correlated with the occurrence of MACEs. The results of multivariate Cox regression analysis were listed in Table 3.

As shown in the Kaplan-Meier curve(see Figure 2), the levels of LY30囚3.0\% significantly increased the risk of MACEs (Log Rank(Mentel-cox): $\chi 2=4.541, P=0.033$ ). When taken LY30 as a categorial variable, multivariate Cox regression analysis indicated that the hazard ratios pertaining to MACEs in patients with LY30 $₫ 0.8 \%$ and $\varangle 3.0 \%$ compared with those in physiologic range(LY30 0.8-3.0\%) were 2.275 [HR: 2.275, 95\% Cl: 1.241-1.241, $\mathrm{P}=0.003$ ] and 1.196 [HR: 1.196, 95\% Cl: 0.679-2.109, $\mathrm{P}=0.535$ ], respectively. Pearson correlation analysis indicated that LY30 was weakly correlated with the level of $A A-I R(r=-0.115 \unrhd p=0.042)$, but not with platelet count, ADP-IR, PT, APTT, fibrinogen and D-dimer( details as shown in Table 4).

\section{Discussion}

Our study observed that more than two thirds of the selected patients with ACS endured abnormal fibrinolysis, which increased the risk of stroke or TIA. Fibrinolysis shutdown was the most common phenotype. However, it is not fibrinolysis shutdown but hyperfibrinolysis (HF) (LY30 >3\%) associated with the occurrence of MACEs in patients with ACS.

The profiles of fibrinolytic dysregulation in patients with ACS were similar as those detected in severely injured patients[22]. In patients with trauma, both fibrinolysis shutdown and hyperfibrinolysis suggest poor prognosis, which indicated a U shaped association with LY30 and mortality[23, 24]. Our study did not exhibit the association between fibrinolysis shutdown and clinical outcomes. Fibrinolysis shutdown represented a hypercoagulable state in previous studies[25], and early antithrombotic therapy might reduce microvascular thrombi and end-organ injury, as well as thromboembolic events. Thanks to early active antithrombotic strategy, fibrinolysis shutdown did not increase the risk of MACEs in patients with ACS. 
D-dimer is a specific byproduct of the enzymatic cleavage of fibrin and its elevation strongly suggests that fibrinolysis is increased. In our univariate analysis, D-dimers and fibrinogen were associated with poor prognosis, but the associations were not significant in multivariate analysis after LY30 was introduced. In our correlation analysis, LY30 was only slightly correlated with AA-IR, but not correlated with D-dimers, fibrinogen and other parameters. Therefore, LY30 might be a more valuable index than Ddimers and other parameters in evaluating the association between prognosis and fibrinolysis.

Our study did not find a relationship between TEG detected platelet inhibition rates and patient's prognosis, although previous platelet mapping assay via modified TEG showed a good correlation with the turbidimetric light transmittance aggregometry, which considered to be the "gold standard" in assessing platelet function[26]. Moreover, small sample study indicated that TEG directed antiplatelet could improve prognosis[27]. However, a randomized study had indicated that TEG directed dual antiplatelet was not benefit for keeping grafts patency and reducing thromboembolic events in patients undergoing coronary artery bypass surgery.[28] Therefore, the potential for clinical applications of TEG in assessing risk of recurrent ischemic events among patients receiving antithrombotic agents remains unclear, but warrants further investigation.

\section{Limitations}

There are several inherent limitations to this study. First, among 2040 patients with CHDs, only 490 of them had TEG test. The patients who receive a TEG test might be more vulnerable to fibrinolytic dysregulation, so there was a selective bias. Second, this retrospective study focused on the process of coagulation and fibrinolysis, but some long-term drug treatment factors, such as $\beta$ blockers, ACEls, ARBs and statins, were not included, which were commonly used in patients with ACS. Third, coagulation, fibrinolysis and bleeding were closely related, only once TEG test could not exhibit the dynamic process, nor to evaluate the benefits of adjusted TEG parameters on clinical outcomes. In addition, due to a limited sample, it is not enough power to assessing the risk of bleeding. Therefore, further studies were needed to answer these unsolved questions.

\section{Conclusions}

Fibrinolysis shutdown was the most common phenotype and likely represents a coagulopathic state in patients with ACS, early antithrombotic therapy might benefit to improve their prognosis. However, hyperfibrinolysis (LY30 >3\%) is still an independent indicator of poor outcomes in patients with ACS, and appropriate clinical interventions still need to explore.

\section{Declarations}

Acknowledgements 
We appreciated the kind supports from coronary care unit and clinical laboratory of Dongguan people's hospital, Southern Medical University.

Authors' contributions

W and DQ. L designed and performed data analysis. HB. W wrote the manuscript. SH. S reviewed the manuscript and supervised the work. All listed authors have read and approved the manuscript.

\section{Funding}

This study was supported by grants from the Natural Science Foundation of Guangdong Province, P.R. China (2017A030313560).

Availability of data and materials

The datasets are available from the corresponding author on reasonable request.

Ethics approval and consent to participate

Not applicable.

Consent for publication

Not applicable.

Competing interests

The authors declare that they have no competing interests.

\section{References}

1. Ibanez B, James S, Agewall S, Antunes MJ, Bucciarelli-Ducci C, Bueno H, Caforio A, Crea F, Goudevenos JA, Halvorsen S et al: 2017 ESC Guidelines for the management of acute myocardial infarction in patients presenting with ST-segment elevation: The Task Force for the management of acute myocardial infarction in patients presenting with ST-segment elevation of the European Society of Cardiology (ESC). EUR HEART J 2018, 39(2):119-177.

2. Valgimigli M, Bueno H, Byrne RA, Collet J, Costa F, Jeppsson A, Juni P, Kastrati A, Kolh P, Mauri L et al: 2017 ESC focused update on dual antiplatelet therapy in coronary artery disease developed in collaboration with EACTS The Task Force for dual antiplatelet therapy in coronary artery disease of the European Society of Cardiology (ESC) and of the European Association for Cardio-Thoracic Surgery (EACTS). European journal of cardio-thoracic surgery: Official journal of the European Association for Cardio-thoracic Surgery 2018, 53(1):34-78.

3. Holcomb JB, Minei KM, Scerbo ML, Radwan ZA, Wade CE, Kozar RA, Gill BS, Albarado R, McNutt MK, Khan $S$ et al: Admission rapid thrombelastography can replace conventional coagulation tests in the 
emergency department: experience with 1974 consecutive trauma patients. ANN SURG 2012, 256(3):476-486.

4. Vogel AM, Radwan ZA, Cox CJ, Cotton BA: Admission rapid thrombelastography delivers real-time "actionable" data in pediatric trauma. J PEDIATR SURG 2013, 48(6):1371-1376.

5. Harahsheh Y, Duff OC, Ho KM: Thromboelastography Predicts Thromboembolism in Critically III Coagulopathic Patients. CRIT CARE MED 2019, 47(6):826-832.

6. Li C, Zhao Q, Yang K, Jiang L, Yu J: Thromboelastography or rotational thromboelastometry for bleeding management in adults undergoing cardiac surgery: a systematic review with meta-analysis and trial sequential analysis. J THORAC DIS 2019, 11(4):1170-1181.

7. Holcomb JB, Minei KM, Scerbo ML, Radwan ZA, Wade CE, Kozar RA, Gill BS, Albarado R, McNutt MK, Khan $S$ et al: Admission rapid thrombelastography can replace conventional coagulation tests in the emergency department: experience with 1974 consecutive trauma patients. ANN SURG 2012, 256(3):476-486.

8. Khalaf-Adeli E, Alavi M, Alizadeh-Ghavidel A, Pourfathollah AA: Comparison of standard coagulation testing with thromboelastometry tests in cardiac surgery. J Cardiovasc Thorac Res 2019, 11(4):300304.

9. Moore HB, Moore EE, Liras IN, Gonzalez E, Harvin JA, Holcomb JB, Sauaia A, Cotton BA: Acute Fibrinolysis Shutdown after Injury Occurs Frequently and Increases Mortality: A Multicenter Evaluation of 2,540 Severely Injured Patients. J Am Coll Surg 2016, 222(4):347-355.

10. Liras IN, Cotton BA, Cardenas JC, Harting MT: Prevalence and impact of admission hyperfibrinolysis in severely injured pediatric trauma patients. SURGERY 2015, 158(3):812-818.

11. Moore HB, Moore EE, Gonzalez E, Chapman MP, Chin TL, Silliman CC, Banerjee A, Sauaia A: Hyperfibrinolysis, physiologic fibrinolysis, and fibrinolysis shutdown: the spectrum of postinjury fibrinolysis and relevance to antifibrinolytic therapy. J Trauma Acute Care Surg 2014, 77(6):811-817, 817.

12. Liras IN, Caplan HW, Stensballe J, Wade CE, Cox CS, Cotton BA: Prevalence and Impact of Admission Acute Traumatic Coagulopathy on Treatment Intensity, Resource Use, and Mortality: An Evaluation of 956 Severely Injured Children and Adolescents. J Am Coll Surg 2017, 224(4):625-632.

13. Peng W, Shi X, Xu X, Lin Y: Both CYP2C19 and PON1 Q192R Genotypes Influence Platelet Response to Clopidogrel by Thrombelastography in Patients with Acute Coronary Syndrome. CARDIOVASC THER 2019, 2019:3470145.

14. A B, RP K: Role of Thrombelastography (TEG) in Risk Assessment and Guidance of Antithrombotic Therapy in Patients with Coronary Artery Disease. DRUG DEVELOP RES 2013, 74(8):533-540.

15. Wang D, Liu Y, Chen Z, Yang F, Zhang Z, Wei Y: Impact of diabetes mellitus on coagulation function before and after off-pump coronary artery bypass grafting. J THORAC DIS 2019, 11(12):5517-5526.

16. Roffi M, Patrono C, Collet JP, Mueller C, Valgimigli M, Andreotti F, Bax JJ, Borger MA, Brotons C, Chew DP et al: 2015 ESC Guidelines for the management of acute coronary syndromes in patients presenting without persistent ST-segment elevation: Task Force for the Management of Acute 
Coronary Syndromes in Patients Presenting without Persistent ST-Segment Elevation of the European Society of Cardiology (ESC). EUR HEART J 2016, 37(3):267-315.

17. 2017 ESCGuidelines for themanagement of acutemyocardial infarction in patients presenting with ST-segment elevation.

18. Moore HB, Moore EE, Liras IN, Gonzalez E, Harvin JA, Holcomb JB, Sauaia A, Cotton BA: Acute Fibrinolysis Shutdown after Injury Occurs Frequently and Increases Mortality: A Multicenter Evaluation of 2,540 Severely Injured Patients. J Am Coll Surg 2016, 222(4):347-355.

19. Liras IN, Cotton BA, Cardenas JC, Harting MT: Prevalence and impact of admission hyperfibrinolysis in severely injured pediatric trauma patients. SURGERY 2015, 158(3):812-818.

20. Moore HB, Moore EE, Gonzalez E, Chapman MP, Chin TL, Silliman CC, Banerjee A, Sauaia A: Hyperfibrinolysis, physiologic fibrinolysis, and fibrinolysis shutdown: the spectrum of postinjury fibrinolysis and relevance to antifibrinolytic therapy. J Trauma Acute Care Surg 2014, 77(6):811-817, 817.

21. Liras IN, Caplan HW, Stensballe J, Wade CE, Cox CS, Cotton BA: Prevalence and Impact of Admission Acute Traumatic Coagulopathy on Treatment Intensity, Resource Use, and Mortality: An Evaluation of 956 Severely Injured Children and Adolescents. J Am Coll Surg 2017, 224(4):625-632.

22. Moore HB, Moore EE, Liras IN, Gonzalez E, Harvin JA, Holcomb JB, Sauaia A, Cotton BA: Acute Fibrinolysis Shutdown after Injury Occurs Frequently and Increases Mortality: A Multicenter Evaluation of 2,540 Severely Injured Patients. J Am Coll Surg 2016, 222(4):347-355.

23. Holcomb JB, Minei KM, Scerbo ML, Radwan ZA, Wade CE, Kozar RA, Gill BS, Albarado R, McNutt MK, Khan $S$ et al: Admission rapid thrombelastography can replace conventional coagulation tests in the emergency department: experience with 1974 consecutive trauma patients. ANN SURG 2012, 256(3):476-486.

24. Vogel AM, Radwan ZA, Cox CJ, Cotton BA: Admission rapid thrombelastography delivers real-time "actionable" data in pediatric trauma. J PEDIATR SURG 2013, 48(6):1371-1376.

25. Moore HB, Moore EE, Liras IN, Gonzalez E, Harvin JA, Holcomb JB, Sauaia A, Cotton BA: Acute Fibrinolysis Shutdown after Injury Occurs Frequently and Increases Mortality: A Multicenter Evaluation of 2,540 Severely Injured Patients. J Am Coll Surg 2016, 222(4):347-355.

26. Bliden KP, DiChiara J, Tantry US, Bassi AK, Chaganti SK, Gurbel PA: Increased risk in patients with high platelet aggregation receiving chronic clopidogrel therapy undergoing percutaneous coronary intervention: is the current antiplatelet therapy adequate? J AM COLL CARDIOL 2007, 49(6):657-666.

27. Xu L, Wang L, Yang X, Li K, Sun H, Zhang D, Wang H, Li W, Ni Z, Xia K et al: Platelet function monitoring guided antiplatelet therapy in patients receiving high-risk coronary interventions. Chin Med J (Engl) 2014, 127(19):3364-3370.

28. Rafiq S, Johansson PI, Kofoed KF, Lund JT, Olsen PS, Bentsen S, Steinbrüchel DA: Thrombelastographic hypercoagulability and antiplatelet therapy after coronary artery bypass surgery (TEG-CABG trial): a randomized controlled trial. PLATELETS 2017, 28(8):786-793. 
Tables

Table 1 The clinical characteristics of enrolled patients with acute coronary syndromes

\begin{tabular}{|c|c|c|c|c|c|}
\hline \multirow[t]{2}{*}{ Variable } & \multirow[t]{2}{*}{ Total } & \multicolumn{3}{|c|}{ Fibrinolysis } & \multirow{2}{*}{$\begin{array}{l}\mathrm{P}- \\
\text { value }\end{array}$} \\
\hline & & Physiologic & Shutdown & Hyper- & \\
\hline $\mathrm{n}$ & 338 & 99 & 163 & 76 & - \\
\hline Males, n(\%) & $272(80.5)$ & $84(30.9)$ & $134(49.3)$ & $54(19.9)$ & 0.055 \\
\hline Age, years & $62.5 \pm 12.8$ & $62.6 \pm 13.3$ & $62.4 \pm 13.1$ & $62.5 \pm 11.6$ & 0.457 \\
\hline Smoking, n(\%) & 199(58.9) & $59(59.6)$ & $97(59.5)$ & $43(56.6)$ & 0.899 \\
\hline Hypertension, n(\%) & $150(44.4)$ & $41(41.4)$ & $70(42.9)$ & $39(51.3)$ & 0.373 \\
\hline Diabetes, n(\%) & $76(22.5)$ & $28(28.3)$ & $30(18.4)$ & $18(23.7)$ & 0.171 \\
\hline STEMI, n(\%) & $137(40.5)$ & $38(38.4)$ & $69(42.3)$ & $30(39.5)$ & 0.801 \\
\hline Stroke, n(\%) & $12(3.6)$ & $3(3.0)$ & $5(3.1)$ & $4(5.3)$ & 0.657 \\
\hline CKD, n(\%) & $22(6.5)$ & $9(9.1)$ & $8(4.9)$ & $5(6.6)$ & 0.412 \\
\hline $\mathrm{CHF}, \mathrm{n}(\%)$ & $11(3.2)$ & $3(3.0)$ & $7(4.3)$ & $1(1.3)$ & 0.476 \\
\hline $\mathrm{SBP}, \mathrm{mmHg}$ & $134.5 \pm 23.9$ & $132.5 \pm 25.8$ & $134.3 \pm 22.7$ & $137.2 \pm 23.9$ & 0.202 \\
\hline Heart ratio, beats/min & $80.6 \pm 16.5$ & $81.0 \pm 18.23$ & $80.5 \pm 16.1$ & $80.4 \pm 15.2$ & 0.254 \\
\hline $\mathrm{BMI}, \mathrm{kg} / \mathrm{m} 2$ & $24.4 \pm 3.5$ & $24.0 \pm 3.0$ & $24.7 \pm 3.9$ & $24.1 \pm 3.1$ & 0.228 \\
\hline Serum Creatine, umol/L & $\begin{array}{l}87.9(71.4- \\
112.9)\end{array}$ & $\begin{array}{l}87.9(73.1- \\
99.4)\end{array}$ & $\begin{array}{l}86.3(70.1- \\
119.5)\end{array}$ & $\begin{array}{l}90.1(72.5- \\
129.0)\end{array}$ & 0.036 \\
\hline ALT, U/L & $31.2(19.3-54.4)$ & $\begin{array}{l}31.2(20.0- \\
54.7)\end{array}$ & $31.8(19.2-54.6)$ & $29(18.8-50.2)$ & 0.762 \\
\hline Leukocytes, $\times 10^{9} / \mathrm{L}$ & $9.8 \pm 3.7$ & $9.6 \pm 3.7$ & $10.0 \pm 3.9$ & $9.4 \pm 3.2$ & 0.244 \\
\hline Hemoglobin, g/L & $129.0 \pm 22.5$ & $130.9 \pm 20.2$ & $130.1 \pm 23.5$ & $124.2 \pm 22.9$ & 0.463 \\
\hline Platelets, $\times 10^{9} / \mathrm{L}$ & $217.7 \pm 66.6$ & $214.1 \pm 69.3$ & $214.9 \pm 63.5$ & $228.3 \pm 69.1$ & 0.637 \\
\hline $\begin{array}{l}\text { Total cholesterol, } \\
\mathrm{mmol} / \mathrm{L}\end{array}$ & $4.8 \pm 1.4$ & $4.7 \pm 1.2$ & $4.7 \pm 1.3$ & $5.1 \pm 1.9$ & 0.026 \\
\hline D-dimers, ug/mL & $0.47(0.28-0.96)$ & $\begin{array}{l}0.48(0.30- \\
1.22)\end{array}$ & $0.47(0.27-0.91)$ & $0.43(0.30-0.85)$ & 0.790 \\
\hline Fibrinogen, g/L & $3.97 \pm 1.27$ & $3.79 \pm 1.00$ & $4.06 \pm 1.46$ & $3.98 \pm 1.13$ & 0.041 \\
\hline APTT, sec & $39.1 \pm 12.8$ & $39.1 \pm 11.7$ & $38.8 \pm 9.9$ & $39.6 \pm 18.6$ & 0.504 \\
\hline PT, sec & $13.6 \pm 3.4$ & $13.2 \pm 1.8$ & $13.9 \pm 4.5$ & $13.4 \pm 1.7$ & 0.104 \\
\hline R-time, $\min$ & $5.53 \pm 2.79$ & $5.13 \pm 2.09$ & $6.04 \pm 3.46$ & $4.98 \pm 1.45$ & 0.003 \\
\hline K-time, min & $1.66 \pm 1.00$ & $1.54 \pm 0.55$ & $1.86 \pm 1.31$ & $1.38 \pm 0.46$ & $\square 0.001$ \\
\hline Angle, degree & $67.41 \pm 8.90$ & $68.32 \pm 6.51$ & $65.5 \pm 10.7$ & $70.28 \pm 5.93$ & $\square 0.001$ \\
\hline $\mathrm{MA}, \mathrm{mm}$ & $64.37 \pm 7.99$ & $64.32 \pm 6.12$ & $64.67 \pm 9.15$ & $63.82 \pm 7.51$ & 0.082 \\
\hline LY30, \% & $0.8(0-2.6)$ & 1.6(1.10-2.10) & $0(0-0.20)$ & $5.05(3.92-7.40)$ & 0.001 \\
\hline ADP-IR, \% & $47.6 \pm 32.8$ & $45.7 \pm 32.9$ & $50.8 \pm 33.1$ & $43.4 \pm 31.7$ & 0.736 \\
\hline AA-IR, \% & $71.2 \pm 28.9$ & $66.6 \pm 32.3$ & $70.4 \pm 28.3$ & $78.4 \pm 24.5$ & 0.009 \\
\hline
\end{tabular}

Notes: STEMI, ST-elevation myocardial infarction; CKD, chronic kidney diseases; CHF, congestive heart failure; SBP, systolic blood pressure; BMI, body mass index =weight(kg)/height(m)2; ALT, alanine aminotransferase; APTT, activated partial thromboplastin time; PT, prothrombin time; MA, maximal amplitude; LY30, lysis 30 minutes after MA; ADP-IR, adenosine diphosphate inhibition rate; AA-IR, arachidonic acid inhibition rate. 
Table2 Clinical outcomes during the follow-up period

\begin{tabular}{llllll}
\hline Events & Total & \multicolumn{3}{c}{ Fibrinolysis } & P value \\
\cline { 3 - 4 } & & Physiologic & Shutdown & Hyper- & \\
& N=338 & N=99 & N=163 & N=76 & \\
\hline MACEs* & $77(22.8)$ & $19(19.2)$ & $33(20.2)$ & $25(32.9)$ & 0.057 \\
All-cause death & $17(5.0)$ & $4(4.0)$ & $10(6.3)$ & $3(3.9)$ & 0.668 \\
ACS & $35(10.4)$ & $9(9.1)$ & $14(8.6)$ & $12(15.8)$ & 0.208 \\
Stroke/TIA & $26(7.7)$ & $3(3.0)$ & $13(8.0)$ & $10(13.2)$ & 0.044 \\
Heart failure & $24(7.1)$ & $4(4.0)$ & $11(6.7)$ & $9(11.8)$ & 0.134 \\
Bleeding & $9(2.7)$ & $4(4.0)$ & $4(2.5)$ & $1(1.3)$ & 0.540 \\
\hline
\end{tabular}

Notes: *ACS, acute coronary syndrome; TIA, transient ischemic attack; MACEs includes all-cause death; ACS, stroke or TIA, and heart failure.

Table 3 Multivariate Cox regression analysis pertaining to MACEs in patients with acute coronary syndrome

\begin{tabular}{llll}
\hline Variable & 1 represents & HR(95\%CI) & P \\
\hline Gender & Male & $1.290 \square 0.700-2.377 \square$ & 0.415 \\
Age & $1-y e a r$ increment & $1.03101 .007-1.056 \square$ & 0.012 \\
SBP & $1 \mathrm{mmHg}$ increment & $1.004 \square 0.995-1.014 \square$ & 0.363 \\
Hemoglobin & $1 \mathrm{~g} / \mathrm{l}$ increment & $0.996(0.981-1.011)$ & 0.573 \\
Serum Creatine & $1 \mathrm{umol} / \mathrm{l}$ increment & $1.001(0.999-1.003)$ & 0.433 \\
D-dimers & $1 \mathrm{ug} / \mathrm{ml}$ increment & $1.067(0.926-1.228)$ & 0.369 \\
Fibrinogen & $1 \mathrm{~g} / \mathrm{lincrement}$ & $1.107(0.923-1.329)$ & 0.273 \\
LY30 & $1 \%$ increment & $1.097(1.013-1.188)$ & 0.023 \\
\hline
\end{tabular}

Notes $\square$ ACS, acute coronary syndrome; HR, hazard ratio; CI, confidence interval; SBP, systolic blood pressure; LY30, lysis 30 minutes after maximal amplitude. MACEs includes all-cause death; ACS, stroke or TIA, and heart failure.

Table 4 Pearson correlation between LY30 and other associated factors.

\begin{tabular}{lll}
\hline Variable & $\mathrm{r}$ & $\mathrm{P}$ value \\
\hline Platelet count & 0.083 & 0.129 \\
ADP-IR & -0.077 & 0.172 \\
AA-IR & -0.115 & 0.042 \\
PT & -0.053 & 0.341 \\
APTT & -0.030 & 0.588 \\
Fibrinogen & -0.020 & 0.717 \\
D-dimers & -0.041 & 0.471 \\
\hline
\end{tabular}

Notes: LY30, lysis 30 minutes after maximal amplitude; ADP-IR, adenosine diphosphate inhibition rate; AA-IR, arachidonic acid inhibition rate; APTT, activated partial thromboplastin time; PT, prothrombin time.

\section{Figures}




\section{Figure 1 Flow diagram of the study}

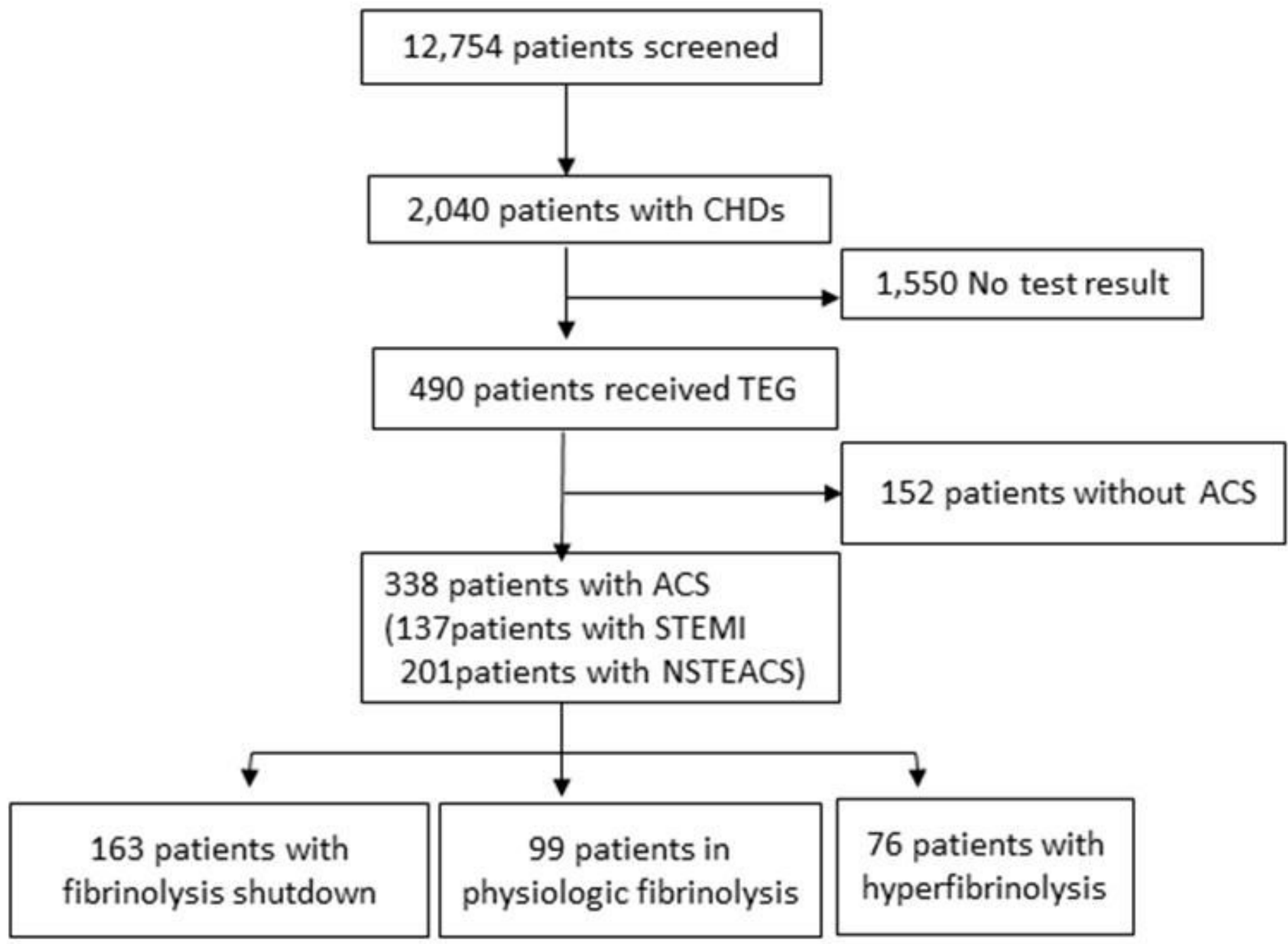

Figure 1

Flow diagram of the study 


\section{Figure 2 Kaplan-meier curve}

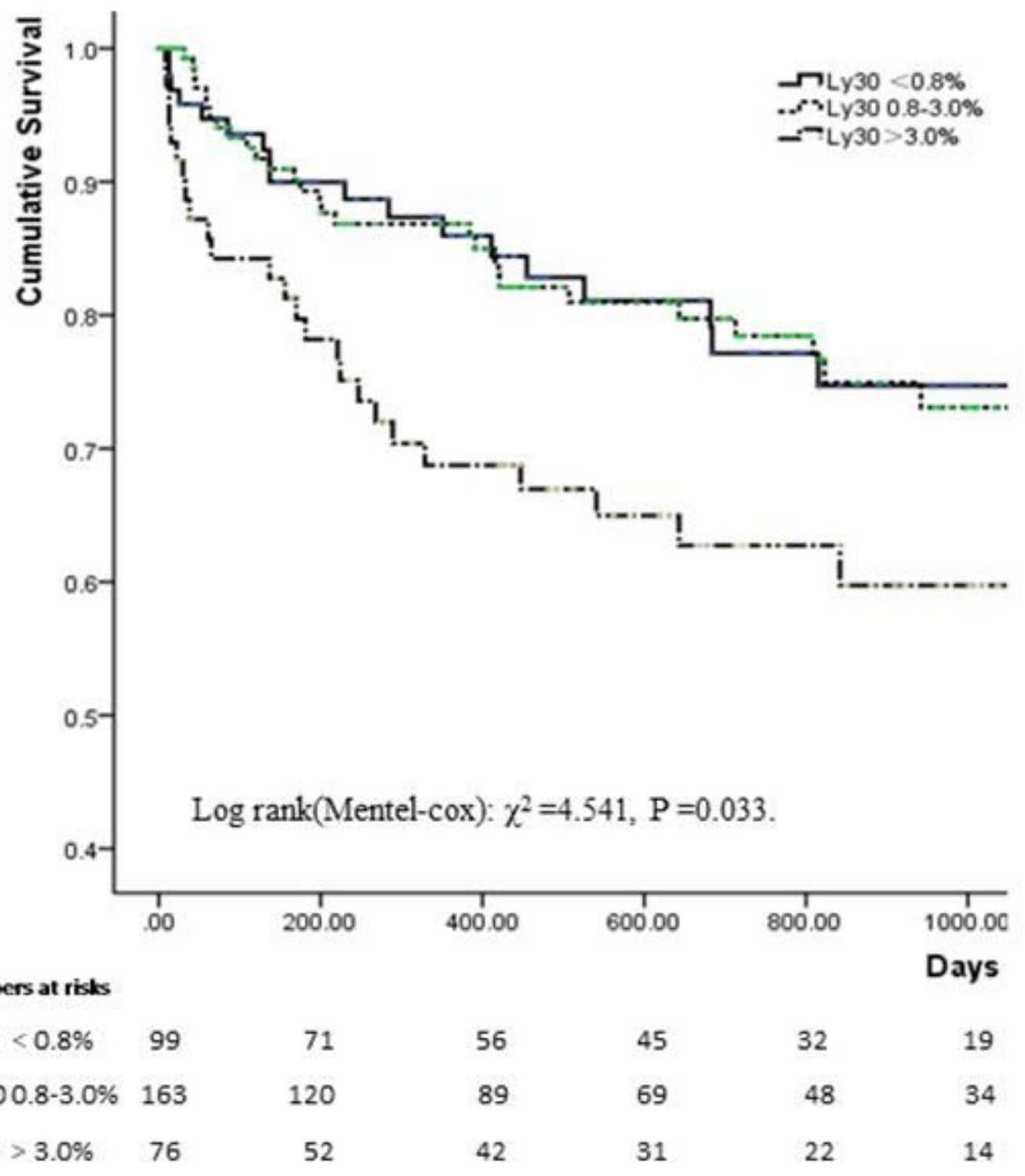

Figure 2

Kaplan-Meier curve 